\section{Epidemiology and Infection}

cambridge.org/hyg

\section{Original Paper}

Cite this article: Asakura K, Nakano M, Omae $K$ (2018). Relationship between bidet toilet use and haemorrhoids and urogenital infections: a 3-year follow-up web survey. Epidemiology and Infection 146, 763-770. https://doi.org/ $10.1017 /$ S0950268818000584

Received: 21 September 2017

Revised: 10 December 2017

Accepted: 16 February 2018

First published online: 21 March 2018

\section{Key words:}

Electric bidet toilet; general population; haemorrhoid; longitudinal study; urogenital infection

Author for correspondence:

K. Asakura, E-mail: JZF01334@nifty.ne.jp

\title{
Relationship between bidet toilet use and haemorrhoids and urogenital infections: a 3-year follow-up web survey
}

K. Asakura ${ }^{1,2}$, M. Nakano ${ }^{2}$ and K. Omae ${ }^{2}$

${ }^{1}$ Department of Environmental and Occupational Health, School of Medicine, Toho University, Tokyo, Japan and ${ }^{2}$ Department of Preventive Medicine and Public Health, School of Medicine, Keio University, Tokyo, Japan

\begin{abstract}
Given the growing use of electric bidet toilets in Japan and other countries, we assessed the relationship between bidet toilet use and haemorrhoids or urogenital infections. Data were collected using a web-based longitudinal survey. In total, 10305 subjects randomly selected from panels of a Japanese website research company for the baseline survey in 2013 were asked about their frequency of bidet toilet use and receipt of a doctor's diagnosis or subjective symptom of haemorrhoids and urogenital infections. One- and three-year follow-up surveys were performed in 2014 and 2016, respectively, and information on newly diagnosed/experienced outcomes occurring during the follow-up period were collected. Cumulative incidence of haemorrhoids and urogenital infections was not significantly increased by habitual use of a bidet toilet. In men, more habitual users reported subjective symptoms of irritated skin around the anus, which were newly experienced during follow-up than non-habitual users (adjusted risk ratio 1.36 (95\% confidence interval 1.06-1.75)). Further studies are needed to confirm this relationship. Several of the outcomes were significantly more prevalent in habitual users, but these results were probably explained by reverse causation.
\end{abstract}

\section{Introduction}

Electric bidet toilets are popular sanitary facilities in Japan. According to the Consumer Confidence Survey of the Government of Japan in 2017, 79.1\% of households have such a toilet [1]. They are becoming popular in other Asian countries such as China, Taiwan and Indonesia [2], and are now also sold in Western countries through electronic commerce companies such as Amazon.com, Inc. [3]. Bidet toilets are used to clean the anal or urogenital area after defecation, urination or during menstruation. They differ from older style bidets in that they consist of an electrically operated washing device, which is housed within the bowl of a normal toilet. Since they were developed as consumer goods, and not as medical instruments, they were not subject to evaluation in clinical trials and their health effects are still under investigation. Water stream from the electric bidet toilets directly wash the anal and urogenital area, and pressure of the stream is stronger than that of the older style bidets. People can also easily wash for longer time because the device can be operated simply by pushing its button, without getting their hands wet. Thus, adverse health effects by too much washing, such as alteration of vaginal microflora, urinary infection or skin damage are concerned.

We previously showed that bidet toilet use was not associated with preterm birth or bacterial vaginosis among pregnant Japanese women [4]. Regarding the general population, we also reported that there was no association between bidet toilet use and haemorrhoids or urogenital infections other than bacterial vaginosis [5], for which no conclusion could be reached owing to the small number of incident cases (37 cases among 3721 women after 1-year follow-up) and the finding of a significant difference in the cumulative incidence based on subjective symptoms without a doctor's diagnosis [5]. Other studies have reported an association between bidet toilet use and pathogenic changes of vaginal microflora [6] or itching of the anus [7].

To better compare the cumulative incidence between bidet toilet users and non-users, we obtained more cases by continuing to follow the same general population enrolled in the previous study [5]. For the present study, we extended the follow-up period to 3 years (baseline survey February 2013; follow-up survey February 2016) and assessed again the relationship between bidet toilet use and haemorrhoids or urogenital infections.

\section{Methods}

\section{Study subjects}

Details of the study design and participant characteristics have been reported elsewhere [5]. Briefly, the study subjects were selected from among approximately 1 million people 
anonymously registered with a leading Japanese website research company's web panel (Macromill, Inc.). A total of 18562 people were randomly selected using a computer programme, to whom a web survey questionnaire was randomly delivered until the number of respondents exceed 10000 . A total of 10305 individuals were involved in the baseline survey, which was conducted in February 2013.

A 1-year follow-up survey was conducted in February 2014 and a 3-year follow-up survey in February 2016. Among the 10 305 subjects involved in the baseline survey, 8225 subjects participated in the 1-year follow-up and 5820 in the 3-year follow-up. To utilise as many subjects as possible, we selected both the subjects who participated in all three surveys (baseline, 1-year, 3 -year) and those who participated in the baseline and either one of two follow-up surveys. In total, 8484 subjects (4170 men and 4314 women) met these selection criteria. Of these, 725 subjects who met exclusion criteria were eliminated from the analysed population, including those who provided inconsistent answers about 'bidet toilet use' at the baseline and follow-up surveys $(n=526)$, were aged over 80 years $(n=98)$, had an abnormal frequency of daily urination $(<3$ or $>15)(n=48)$ or abnormal hours of daily sleep $(<4 \mathrm{~h})(n=24)$, as well as females older than 60 years with current menstruation $(n=23)$, and those with unusually prolonged bidet toilet use $(>180 \mathrm{~s})(n=6)$. These exclusion criteria matched those used in the 1-year follow-up study [5]. The final number of subjects analysed was 7759 , giving a follow-up rate of $75.3 \%(7759 / 10305 \times 100)$. The website research company was unable to send the web research form to 1964 subjects during the 3-year follow-up, probably due to their withdrawal from registration as members of the web panel. Since this loss to follow-up occurred irrespective of the present study, the true participation rate would be higher than the simple follow-up rate shown above.

\section{Questionnaire survey}

Information about bidet toilet use was collected using selfadministered questionnaires through the web survey system of Macromill, Inc. in both baseline and two follow-up surveys. The question on the frequency of bidet toilet use was answered using three exposure levels, namely 'never', '<once a week' and 'every day or $\geqslant$ once a week'. Based on their answer to this question, the subjects were categorised into two groups: 'habitual users', who used a bidet toilet $\geqslant$ once a week, and 'non-habitual users', who used one <once a week or never.

Outcomes for both sexes were haemorrhoids and irritated perianal skin. For women, cystitis, pyelonephritis, candida vaginitis, bacterial vaginosis and vulval pruritus were also assessed as health outcomes. Physician diagnoses and subjective symptoms of these outcomes were analysed separately. Subjective symptoms of these diseases are highly specific, and usually used as important clues in the diagnosis of these diseases. To avoid misunderstanding or confusion of the outcome questions, signs and symptoms of the outcomes were displayed on the same screen as the questions. We asked about past history at the baseline survey. In the follow-up survey, we requested the subjects to answer a question asking whether the diseases (or symptoms) were 'newly diagnosed' (or newly experienced) during the period from the baseline survey to the follow-up surveys (February 2013 to February 2014 in the 1-year follow-up, and February 2013 to February 2016 in the 3-year follow-up survey); 'ever diagnosed' (or ever experienced) before the baseline survey; or 'never diagnosed' (or never experienced). If the subject answered 'newly diagnosed' (or newly experienced), we counted him/her as an incident case, and if he/she answered 'ever diagnosed' (or ever experienced), we counted him/her as a prevalent case. If a subject answered either of two follow-up surveys, the existing answer was used to define the outcome status. If a subject answered both of the follow-up surveys and the answers were inconsistent, he/she was not included in the analysis for the corresponding diseases or symptoms. Exceptionally, subjects who answered 'never diagnosed' in the 1-year follow-up survey and 'newly diagnosed' in the 3-year follow-up survey were included in the analysis as incident cases.

The questionnaire included questions about educational background, smoking, drinking, fitness, sleeping, showering/bathing, bowel movements, direction of wiping the anus after defecation, menstrual status, sexual activity, past/current histories of diseases and medication use. Basic characteristics of the subjects such as age, sex, residential area, marital status, household income, etc., were already registered in the website research company records and were provided to us.

\section{Statistical analysis}

The prevalence and cumulative incidence of outcomes were compared between habitual and non-habitual users by Poisson regression analysis with robust standard errors, and risk ratios (RRs) were shown. Covariates for the multivariate models were harmonised with the previous study showing the results of 1 -year follow up [5]. In detail, age category (20-39/40-59/60-79 years old), marital status, educational background (low (senior high school or lower)/high (junior college or higher)), smoking habits (current/former/never), alcohol drinking habits (habitual/occasional/ no), current history of immune-suppressing diseases (yes/no) and current constipation (yes/no) were included in the analysis of haemorrhoids and related outcomes; and age category, marital status, smoking habit, current menstrual status (yes/no), sexual activity (yes/no) and current constipation (yes/no) were included in the analysis of urogenital outcomes in women.

Statistical significance was assessed by a two-tailed analysis. The level of significance adopted was 5\%. All statistical analyses were performed using commercial software (SAS version 9.4; SAS Institute, Cary NC, USA).

\section{Results}

The characteristics of the analysed subjects are shown in Table 1. The number of habitual bidet toilet users was 3889 (50.1\% of all subjects), and that of non-habitual users was 3870 (49.9\%). By sex, the proportion of habitual users was $51.8 \%$ in men and $48.5 \%$ in women. Distributions of most factors shown in Table 1 were significantly different between the habitual and nonhabitual users. For example, habitual users were older, likely to be married and wealthier.

The RRs of haemorrhoids diagnosed by a physician, subjective symptoms of haemorrhoids and subjective symptoms of irritated perianal skin are shown in Table 2 by sex. The RRs were calculated based on the prevalence and cumulative incidence. All RRs based on the prevalence showed significantly higher prevalence of these three outcomes in habitual users. In contrast, the only one adjusted RR based on the cumulative incidence, that for the subjective symptom of irritated skin around the anus, showed a significantly higher incidence in male habitual users 
Table 1. Characteristics of subjects $(n=7759)$

\begin{tabular}{|c|c|c|c|c|c|c|c|c|c|c|c|c|}
\hline \multirow{2}{*}{ Habitual use of bidet toilet } & \multicolumn{4}{|c|}{ All $(n=7759)$} & \multicolumn{4}{|c|}{ Women $(n=3929)$} & \multicolumn{4}{|c|}{ Men $(n=3830)$} \\
\hline & \multicolumn{2}{|c|}{ No $(n=3870)$} & \multicolumn{2}{|c|}{ Yes $(n=3889)$} & \multicolumn{2}{|c|}{ No $(n=2025)$} & \multicolumn{2}{|c|}{ Yes $(n=1904)$} & \multicolumn{2}{|c|}{ No $(n=1845)$} & \multicolumn{2}{|c|}{ Yes $(n=1985)$} \\
\hline \multicolumn{13}{|l|}{ Age (years) } \\
\hline 20-39 & 1398 & 36.1 & 608 & 15.6 & 702 & 34.7 & 240 & 12.6 & 696 & 37.7 & 368 & 18.5 \\
\hline $60-79$ & 1115 & 28.8 & 2068 & 53.2 & 643 & 31.8 & 1087 & 57.1 & 472 & 25.6 & 981 & 49.4 \\
\hline \multicolumn{13}{|l|}{ Marital status } \\
\hline Unmarried & 1527 & 39.5 & 889 & 22.9 & 703 & 34.7 & 444 & 23.3 & 824 & 44.7 & 445 & 22.4 \\
\hline Married & 2343 & 60.5 & 3000 & 77.1 & 1322 & 65.3 & 1460 & 76.7 & 1021 & 55.3 & 1540 & 77.6 \\
\hline \multicolumn{13}{|l|}{ Educational background ${ }^{\mathrm{a}, \mathrm{b}}$} \\
\hline \multicolumn{13}{|c|}{ Household income $^{\mathrm{b}}$ (million yen/year) } \\
\hline$<4$ & 1355 & 41.9 & 1163 & 33.7 & 723 & 44.2 & 616 & 37.4 & 632 & 39.6 & 547 & 30.3 \\
\hline 4 to $<8$ & 1324 & 40.9 & 1573 & 45.6 & 663 & 40.5 & 727 & 44.1 & 661 & 41.4 & 846 & 46.9 \\
\hline$\geqslant 8$ & 555 & 17.2 & 715 & 20.7 & 250 & 15.3 & 305 & 18.5 & 305 & 19.1 & 410 & 22.7 \\
\hline \multicolumn{13}{|l|}{ Cigarette smoking } \\
\hline Never & 2591 & 67.0 & 2568 & 66.0 & 1612 & 79.6 & 1582 & 83.1 & 979 & 53.1 & 986 & 49.7 \\
\hline Former & 516 & 13.3 & 716 & 18.4 & 165 & 8.2 & 149 & 7.8 & 351 & 19.0 & 567 & 28.6 \\
\hline Current & 763 & 19.7 & 605 & 15.6 & 248 & 12.3 & 173 & 9.1 & 515 & 27.9 & 432 & 21.8 \\
\hline Yes & 399 & 10.3 & 661 & 17.0 & 174 & 8.6 & 301 & 15.8 & 225 & 12.2 & 360 & 18.1 \\
\hline \multicolumn{13}{|c|}{ Frequency of showering/bathing ${ }^{\mathrm{b}}$} \\
\hline$\geqslant$ Once/day & 3034 & 81.5 & 2959 & 78.9 & 1622 & 82.8 & 1449 & 79.6 & 1412 & 80.1 & 1510 & 78.3 \\
\hline$<$ Once/day & 687 & 18.5 & 791 & 21.1 & 337 & 17.2 & 372 & 20.4 & 350 & 19.9 & 419 & 21.7 \\
\hline \multicolumn{13}{|l|}{ Current constipation } \\
\hline No & 2021 & 52.2 & 2229 & 57.3 & 860 & 42.5 & 938 & 49.3 & 1161 & 62.9 & 1291 & 65.0 \\
\hline Yes & 1849 & 47.8 & 1660 & 42.7 & 1165 & 57.5 & 966 & 50.7 & 684 & 37.1 & 694 & 35.0 \\
\hline \multicolumn{13}{|l|}{ Current menstruation $^{\mathrm{b}}$} \\
\hline Yes & - & - & - & - & 1143 & 56.6 & 524 & 27.7 & - & - & - & - \\
\hline No & - & - & - & - & 876 & 43.4 & 1371 & 72.4 & - & - & - & - \\
\hline \multicolumn{13}{|c|}{ Direction of wiping anus after defecation ${ }^{\text {b }}$} \\
\hline Front to back & - & - & - & - & 1233 & 62.8 & 1089 & 66.3 & - & - & - & - \\
\hline Back to front & - & - & - & - & 729 & 37.2 & 553 & 33.7 & - & - & - & - \\
\hline Sexual activity $(\geqslant o n c e / \text { year })^{b}$ & & & & & & & & & & & & \\
\hline No & - & - & - & - & 1142 & 60.7 & 1301 & 71.8 & - & - & - & - \\
\hline Yes & - & - & - & - & 739 & 39.3 & 511 & 28.2 & - & - & - & - \\
\hline
\end{tabular}

aLow means graduation from high school or lower schools. High means graduation from a junior college or higher educational institution.

'Some subjects did not answer the questions or selected 'unknown'. Number of such subjects were 50 in 'Educational background', 1074 in 'Household income', 288 in 'Frequency of showering/bathing', 15 in 'Current menstruation', 325 in 'Direction of wiping anus after defecation' and 236 in 'Sexual activity'.

'Immune-suppressing diseases include diabetes, malignancy and immune disorder. 
Table 2. Prevalence, cumulative incidence and risk ratios of haemorrhoids and related symptoms in habitual bidet toilet users and non-habitual users

\begin{tabular}{|c|c|c|c|c|c|c|c|c|c|c|c|c|c|c|c|c|}
\hline & \multicolumn{4}{|c|}{$\begin{array}{c}\text { Number of subjects who have been/ } \\
\text { have never been diagnosed or } \\
\text { experienced symptoms before } \\
\text { survey }\end{array}$} & \multicolumn{4}{|c|}{ Risk ratio based on prevalence } & \multicolumn{4}{|c|}{$\begin{array}{l}\text { Number of subjects who were/were } \\
\text { not newly diagnosed or experienced } \\
\text { symptoms during follow-up }\end{array}$} & \multicolumn{4}{|c|}{ Risk ratio based on cumulative incidence } \\
\hline & Yes & $(\%)$ & No & $(\%)$ & \multicolumn{2}{|c|}{ Crude $(95 \% \mathrm{Cl})$} & \multicolumn{2}{|c|}{ Adjusted $(95 \% \mathrm{Cl})^{\mathrm{a}}$} & Yes & $(\%)$ & $\mathrm{No}^{\mathrm{b}}$ & $(\%)$ & \multicolumn{2}{|c|}{ Crude $(95 \% \mathrm{Cl})$} & \multicolumn{2}{|c|}{ Adjusted $(95 \% \mathrm{CI})^{\mathrm{a}}$} \\
\hline \multicolumn{17}{|c|}{ Doctor's diagnosis of haemorrhoids } \\
\hline \multicolumn{17}{|l|}{ Men } \\
\hline Habitual user ${ }^{c}$ & 500 & $(27.1)$ & 1344 & (72.9) & 2.14 & $(1.86-2.47)$ & 1.69 & $(1.45-1.96)$ & 27 & $(2.0)$ & 1317 & (98.0) & 1.55 & $(0.87-2.74)$ & 1.67 & $(0.90-3.10)$ \\
\hline Non-habitual user & 223 & (12.7) & 1539 & (87.3) & ref & & ref & & 20 & $(1.3)$ & 1519 & (98.7) & ref & & ref & \\
\hline \multicolumn{17}{|l|}{ Women } \\
\hline Habitual user $^{c}$ & 315 & $(17.6)$ & 1478 & $(82.4)$ & 1.52 & $(1.30-1.78)$ & 1.55 & $(1.31-1.83)$ & 21 & $(1.4)$ & 1457 & (98.6) & 0.88 & $(0.50-1.54)$ & 1.12 & $(0.62-2.03)$ \\
\hline Non-habitual user & 226 & (11.6) & 1726 & (88.4) & ref & & ref & & 28 & $(1.6)$ & 1698 & (98.4) & ref & & ref & \\
\hline \multicolumn{17}{|c|}{ Subjective symptom of haemorrhoids } \\
\hline \multicolumn{17}{|l|}{ Men } \\
\hline Habitual user $^{c}$ & 994 & $(58.3)$ & 711 & $(41.7)$ & 1.56 & $(1.45-1.69)$ & 1.47 & $(1.36-1.59)$ & 59 & $(8.3)$ & 652 & (91.7) & 0.98 & $(0.71-1.35)$ & 1.18 & $(0.86-1.61)$ \\
\hline Non-habitual user & 589 & $(37.3)$ & 992 & $(62.8)$ & ref & & ref & & 84 & (8.5) & 908 & $(91.5)$ & ref & & ref & \\
\hline \multicolumn{17}{|l|}{ Women } \\
\hline Habitual user $^{\mathrm{c}}$ & 746 & $(44.9)$ & 917 & $(55.1)$ & 1.12 & $(1.04-1.21)$ & 1.19 & $(1.10-1.29)$ & 49 & $(5.3)$ & 868 & $(94.7)$ & 0.66 & $(0.47-0.92)$ & 1.13 & $(0.78-1.62)$ \\
\hline Non-habitual user & 721 & $(40.0)$ & 1080 & $(60.0)$ & ref & & ref & & 88 & $(8.2)$ & 992 & $(91.9)$ & ref & & ref & \\
\hline \multicolumn{17}{|c|}{ Subjective symptom of irritated skin around anus } \\
\hline \multicolumn{17}{|l|}{ Men } \\
\hline Habitual user $^{\mathrm{c}}$ & 803 & $(52.5)$ & 727 & $(47.5)$ & 1.51 & $(1.39-1.65)$ & 1.48 & $(1.35-1.61)$ & 100 & $(13.8)$ & 627 & $(86.2)$ & 1.18 & $(0.92-1.52)$ & 1.36 & $(1.06-1.75)$ \\
\hline Non-habitual user & 521 & $(34.7)$ & 981 & (65.3) & ref & & ref & & 114 & $(11.6)$ & 867 & $(88.4)$ & ref & & ref & \\
\hline \multicolumn{17}{|l|}{ Women } \\
\hline Habitual user $^{c}$ & 609 & $(40.2)$ & 906 & $(59.8)$ & 1.17 & $(1.07-1.28)$ & 1.23 & $(1.12-1.35)$ & 100 & $(11.0)$ & 806 & $(89.0)$ & 0.86 & $(0.68-1.09)$ & 1.14 & $(0.89-1.46)$ \\
\hline Non-habitual user & 578 & (34.3) & 1107 & (65.7) & ref & & ref & & 142 & (12.8) & 965 & $(87.2)$ & ref & & ref & \\
\hline
\end{tabular}

$95 \% \mathrm{Cl}, 95 \%$ confidence interval; ref, reference group.

Risk ratio was calculated by Poisson regression models with robust standard errors. The models used to calculate adjusted risk ratios included the confounders of age category (20-39/40-59/60-79 years old), marital status (unmarried/married),

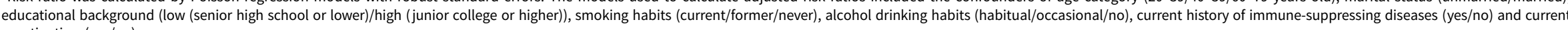
constipation (yes/no).

Numbers of subjects who were not newly diagnosed or who did not experience the outcomes included those who had never been diagnosed or had never experienced them. In other words, those who answered that they had ever been diagnosed or had ever experienced them before the baseline survey were excluded from the analysis.

CHabitual users means those who used the bidet toilet $\geqslant$ once a week. 
Table 3. Prevalence, cumulative incidence and risk ratios of urogenital outcomes in female habitual bidet toilet users and female non-habitual users

\begin{tabular}{|c|c|c|c|c|c|c|c|c|c|c|c|c|c|c|c|c|}
\hline & \multicolumn{4}{|c|}{$\begin{array}{l}\text { Number of subjects who have } \\
\text { been/have never been diagnosed or } \\
\text { experienced symptoms before } \\
\text { survey }\end{array}$} & \multicolumn{4}{|c|}{ Risk ratio based on prevalence } & \multicolumn{4}{|c|}{$\begin{array}{l}\text { Number of subjects who were/ } \\
\text { were not newly diagnosed or } \\
\text { experienced symptoms during } \\
\text { follow-up }\end{array}$} & \multicolumn{4}{|c|}{ Risk ratio based on cumulative incidence } \\
\hline & Yes & $(\%)$ & No & $(\%)$ & \multicolumn{2}{|c|}{ Crude $(95 \% \mathrm{Cl})$} & \multicolumn{2}{|c|}{ Adjusted $(95 \% \mathrm{Cl})^{\mathrm{a}}$} & Yes & $(\%)$ & $\mathrm{No}^{\mathrm{b}}$ & (\%) & \multicolumn{2}{|c|}{ Crude $(95 \% \mathrm{Cl})$} & \multicolumn{2}{|c|}{ Adjusted $(95 \% \mathrm{Cl})^{\mathrm{a}}$} \\
\hline \multicolumn{17}{|l|}{ Doctor's diagnosis of: } \\
\hline \multicolumn{17}{|l|}{ Cystitis } \\
\hline Habitual user ${ }^{c}$ & 683 & $(39.0)$ & 1067 & $(61.0)$ & 1.38 & $(1.26-1.52)$ & 1.20 & $(1.09-1.33)$ & 34 & $(3.2)$ & 1033 & $(96.8)$ & 1.26 & $(0.79-2.02)$ & 1.21 & $(0.73-2.01)$ \\
\hline Non-habitual user & 530 & $(28.2)$ & 1347 & (71.8) & ref & & ref & & 34 & $(2.5)$ & 1313 & $(97.5)$ & ref & & ref & \\
\hline \multicolumn{17}{|l|}{ Pyelonephritis } \\
\hline Habitual user ${ }^{c}$ & 146 & (7.9) & 1704 & (92.1) & 1.43 & $(1.13-1.82)$ & 1.23 & $(0.95-1.59)$ & 6 & $(0.4)$ & 1698 & (99.7) & 0.60 & $(0.22-1.62)$ & 0.67 & $(0.24-1.87)$ \\
\hline Non-habitual user & 109 & (5.5) & 1872 & (94.5) & ref & & ref & & 11 & $(0.6)$ & 1861 & (99.4) & ref & & ref & \\
\hline \multicolumn{17}{|l|}{ Candida vaginitis } \\
\hline Habitual user ${ }^{c}$ & 329 & $(18.5)$ & 1452 & $(81.5)$ & 1.16 & $(1.01-1.34)$ & 1.24 & $(1.07-1.44)$ & 12 & $(0.8)$ & 1440 & (99.2) & 0.45 & $(0.23-0.87)$ & 0.90 & $(0.44-1.87)$ \\
\hline Non-habitual user & 307 & $(15.9)$ & 1621 & $(84.1)$ & ref & & ref & & 30 & (1.9) & 1591 & $(98.2)$ & ref & & ref & \\
\hline \multicolumn{17}{|l|}{ Bacterial vaginosis } \\
\hline Habitual user ${ }^{c}$ & 19 & $(1.0)$ & 1846 & (99.0) & 0.88 & $(0.48-1.61)$ & 1.34 & $(0.70-2.59)$ & 5 & $(0.3)$ & 1841 & (99.7) & 0.48 & $(0.17-1.39)$ & 0.86 & $(0.28-2.68)$ \\
\hline Non-habitual user & 23 & (1.2) & 1967 & (98.8) & ref & & ref & & 11 & $(0.6)$ & 1956 & (99.4) & ref & & ref & \\
\hline \multicolumn{17}{|l|}{ Vulval pruritus } \\
\hline Habitual user ${ }^{c}$ & 18 & $(1.0)$ & 1839 & $(99.0)$ & 0.92 & $(0.49-1.72)$ & 0.71 & $(0.36-1.39)$ & 8 & $(0.4)$ & 1831 & (99.6) & 0.95 & $(0.37-2.47)$ & 0.77 & $(0.29-2.04)$ \\
\hline Non-habitual user & 21 & (1.1) & 1975 & (99.0) & ref & & ref & & 9 & $(0.5)$ & 1966 & (99.5) & ref & & ref & \\
\hline \multicolumn{17}{|l|}{ Subjective symptom of: } \\
\hline \multicolumn{17}{|l|}{ Cystitis } \\
\hline Habitual user ${ }^{c}$ & 752 & $(44.0)$ & 958 & $(56.0)$ & 1.32 & $(1.21-1.43)$ & 1.18 & $(1.08-1.29)$ & 52 & $(5.4)$ & 906 & (94.6) & 1.10 & $(0.76-1.57)$ & 1.30 & $(0.89-1.91)$ \\
\hline Non-habitual user & 608 & (33.4) & 1211 & (66.6) & ref & & ref & & 60 & $(5.0)$ & 1151 & (95.1) & ref & & ref & \\
\hline \multicolumn{17}{|l|}{ Pyelonephritis } \\
\hline Habitual user ${ }^{c}$ & 113 & (6.2) & 1721 & (93.8) & 1.52 & $(1.15-2.01)$ & 1.27 & $(0.94-1.72)$ & 3 & $(0.2)$ & 1718 & $(99.8)$ & 0.30 & $(0.08-1.07)$ & 0.38 & $(0.11-1.35)$ \\
\hline Non-habitual user & 80 & (4.1) & 1891 & (95.9) & ref & & ref & & 11 & $(0.6)$ & 1880 & (99.4) & ref & & ref & \\
\hline \multicolumn{17}{|l|}{ Candida vaginitis } \\
\hline Habitual user ${ }^{c}$ & 356 & $(20.6)$ & 1373 & (79.4) & 1.11 & $(0.97-1.27)$ & 1.23 & $(1.07-1.41)$ & 16 & $(1.2)$ & 1357 & $(98.8)$ & 0.49 & $(0.28-0.88)$ & 1.07 & $(0.56-2.02)$ \\
\hline Non-habitual user & 347 & $(18.6)$ & 1524 & (81.5) & ref & & ref & & 36 & $(2.4)$ & 1488 & (97.6) & ref & & ref & \\
\hline \multicolumn{17}{|l|}{ Bacterial vaginosis } \\
\hline Habitual user ${ }^{c}$ & 36 & (2.0) & 1807 & (98.1) & 1.20 & $(0.75-1.93)$ & 1.47 & $(0.87-2.48)$ & 16 & $(0.9)$ & 1791 & (99.1) & 0.90 & $(0.47-1.75)$ & 1.37 & $(0.67-2.81)$ \\
\hline
\end{tabular}

Cystitis 


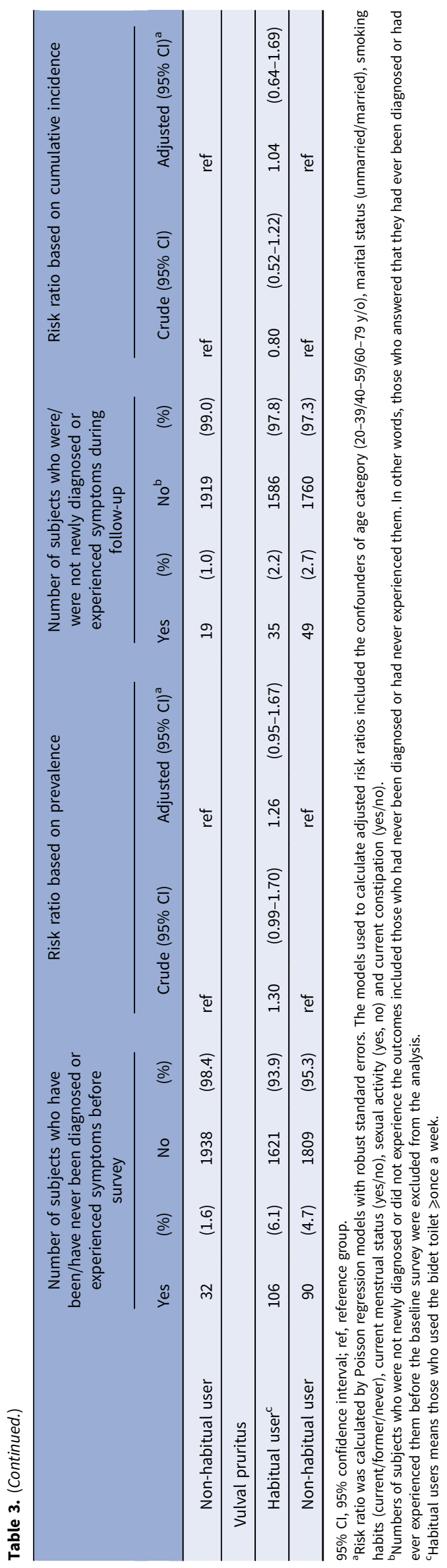

(1.36, 95\% confidence interval (CI) 1.06-1.75). The other RRs based on the cumulative incidence did not significantly differ.

Table 3 shows the RRs of urogenital outcomes diagnosed by a physician and subjective symptoms of the outcomes in women. RRs of cystitis ever diagnosed by a physician and ever reported by a subject herself were higher in the habitual users (adjusted RRs (95\% CI) 1.20 (1.09-1.33) for diagnosed cases, 1.18 (1.081.29) for self-reported cases). However, the RRs based on the cumulative incidence implied that almost the same proportion of habitual and non-habitual users were newly diagnosed or experienced this condition. The crude RR based on the prevalence for pyelonephritis (both diagnosed and self-reported) showed a significantly higher prevalence in habitual users, but the adjusted RR based on the prevalence and the RRs based on the cumulative incidence showed only a non-significant difference between the habitual and non-habitual users. Regarding candida vaginitis, both diagnosed by a physician and self-reported, the adjusted RRs based on the prevalence implied that the prevalence was significantly higher in the habitual users, whereas the crude RRs based on the cumulative incidence showed the opposite association, namely a significantly lower incidence in habitual users.

\section{Discussion}

The cumulative incidences of most outcomes assessed in this study did not show an association with habitual use of a bidet toilet. In men, the cumulative incidence of the subjective symptom of irritated skin around the anus was significantly higher in habitual users. Regarding women, no cumulative incidence of outcomes was significantly associated with bidet toilet use. To the contrary, the prevalence of haemorrhoids and related symptoms was significantly higher in habitual users among both men and women. The prevalence of many urogenital outcomes in female subjects was also significantly higher in the habitual users. These different results between the cumulative incidence and the prevalence are probably explained by reverse causation, as we stated in our previous study [5]. For example, those who suffered haemorrhoids may habitually use the bidet toilet to alleviate symptoms such as pain or discomfort around the anus.

We followed the same population as in our previous study, which showed the results of 1-year follow-up, for an additional 2 more years [5]. In that study, the cumulative incidence of bacterial vaginosis was significantly higher in female habitual users, but a conclusive result could not be obtained because the incidence rate was too low [5]. This measure did not differ between habitual and non-habitual users in the present study. Although the cumulative incidence was still small, these findings may indicate that a strong relationship between bacterial vaginosis and bidet toilet use does not exist. In contrast, the subjective symptom of irritated skin around the anus was significantly related with bidet toilet use among men in the present study only. Men who reported this symptom were younger and more constipated, and reported a shorter period of bidet toilet use and both less frequent and briefer washing of the anus (data not shown). Tsunoda et al. reported that $30 \%$ of bidet toilet users washed the anus before defecation [7]. Bidet toilet users who were usually constipated tended to wash the anus before defecation and preferred a stronger water jet [7]. The subjects who newly experienced irritated skin around the anus in the present study were clight users (use for a shorter period, less frequent use and shorter time for each use)' based on our data, but we did not ask about water jet pressure in our survey. It is possible that constipated, young men who 
use the bidet toilet before defecation tend to experience (or care about) irritated skin around the anus. Further studies are needed to confirm whether or not this relationship was due to chance.

The period of follow-up was extended to obtain more incident cases and to allow cumulative incidences to be more efficiently compared between habitual and non-habitual users. However, the number of incident cases did not increase as expected. At the time of the follow-up surveys, we asked the subjects whether they were newly diagnosed or newly experienced each outcome during the preceding 1 (1-year follow-up) or 3 (3-year follow-up) years. Memories of health-related episodes occurring some years previously might be difficult to recall in detail, and a number of incident cases reported in the 1-year follow-up survey might have been experienced relatively recently, but still more than 1 year before. From a different perspective, the answers given by each subject remained stable between the 1- and 3-year follow-up surveys. The concordance of answers between the 1- and 3-year follow-ups likely supports the stability of the answers from the subjects. The proportion of matched answers for the diagnosis of haemorrhoids was $91.3 \%$ in men and $92.3 \%$ in women. Of note, the subjects were not provided information about their past answers in the follow-up surveys, and any subject whose answers in the two follow-up surveys did not match was excluded from the analysis.

To date, a few studies have examined the effect of bidet toilet use on health. Ogino et al. reported that the habitual use of a bidet toilet promotes pathogenic changes of vaginal microflora [6], while Kohdaira showed that women who experienced recurrent cystitis more frequently used the bidet toilet [8]. However, these two studies did not consider the possibility of reverse causation. Their studies were hospital-based and included female patients with subjective symptoms. Given our finding that $10.7 \%$ of female bidet toilet users had started using a bidet toilet to mitigate gynaecologic discomfort [4], it is natural that the proportion of bidet toilet users is higher in those with subjective urogenital symptoms than in those without. Our present study, which took an account of reverse causation, suggested that active use of a bidet toilet may be associated with itching of the anus in the general population [7]. Extreme use, namely use that is too frequent, too long or with too strong a jet, is better avoided.

The data for the present study were collected through a webbased survey. As discussed in our previous study [5], this kind of study has advantages and disadvantages compared with traditional studies. One disadvantage is the representativeness of the subjects. Some studies have shown that study subjects recruited by Internet-based approaches generally tend to be younger and to have a higher socio-economic status than traditional, non-Internet-based subjects [9-11]. The population analysed in the present study included more old people than the general Japanese population, and this may reflect a high usage rate of bidet toilets and greater interest in this facility among older people. Given that the proportion of high school graduates who entered junior colleges or universities in 1990 was 30.5\% [12], our present subjects appear more highly educated than the general population. Although we should mention that the representativeness of our subjects was not ideal, this kind of deviation is not specific to web-based surveys. Our consideration of factors such as age and socio-economic status in the analysis meant that our comparison of habitual and non-habitual users was performed appropriately. Another concern about the web-based survey may be the possibility of incorrect answers due to anonymity. However, van Gelder et al. reported that the anonymity of web- based surveys is rather an advantage: information about traditional epidemiologic risk factors can be collected with equal or even better reliability due to the anonymity and privacy of response they afford [13]. Because our survey included questions about urogenital symptoms, this anonymity is most likely to have benefitted the accuracy of answers. In addition, the electronic data handling process averts errors in data entry and the coding process.

Our study has other strengths and limitations irrespective of the use of the Internet. We gave particular consideration to reverse causality by employing a prospective design and recruiting general people, rather than patients or those with symptoms. On the other hand, inconsistency of answers in the three surveys hindered the classification of exposure and outcome status in each subject, and in fact we had to exclude approximately $15 \%$ of subjects from the analysis due to this inconsistency in exposure and/ or outcomes.

In conclusion, we found that haemorrhoids and urogenital infections were not associated with habitual bidet toilet use. More men who habitually used a bidet toilet experienced the subjective symptom of irritated skin around the anus. Further studies are needed to confirm this relationship. It is possible that the unusual use of a bidet toilet, such as a stimulant for defecation, may cause skin irritation around the anus.

Acknowledgement. This work was supported by the Japan Sanitary Equipment Industry Association (JSEIA). JSEIA had no role in the design of the study, the collection and analysis of the data, or the preparation of the manuscript.

Conflict of interest. This study was sponsored by JSEIA.

Ethical standards. The authors assert that all procedures contributing to this work comply with the ethical standards of the relevant national and institutional committees on human experimentation and with the Helsinki Declaration of 1975, as revised in 2008. This study was approved by the Ethics Committee, Keio University School of Medicine (approval number: 20120410)

\section{References}

1. Cabinet Office, Government of Japan. Consumer Confidence Survey 2017. Available at http://www.esri.cao.go.jp/jp/stat/shouhi/shouhi.html\#taikyuu (Accessed 20 September 2017) (in Japanese).

2. Inoue K. IT media executive. Available at http://mag.executive.itmedia.co. jp/executive/articles/1303/13/news012.html (Accessed 20 September 2017) (in Japanese).

3. Amazon.com, Inc. Amazon Best Sellers. Available at https://www.amazon. com/Best-Sellers-Home-Improvement-Bidet-Seats/zgbs/hi/6810566011 (Accessed 20 September 2017).

4. Asakura K, et al. (2013) Effect of bidet toilet use on preterm birth and vaginal flora in pregnant women. Obstetrics and Gynecology 121, 11871194.

5. Kiuchi T, et al. (2017) Bidet toilet use and incidence of hemorrhoids or urogenital infections: a one-year follow-up web survey. Preventive Medicine Reports 6, 121-125.

6. Ogino M, Iino K and Minoura S (2010) Habitual use of warm-water cleaning toilets is related to the aggravation of vaginal microflora. The Journal of Obstetrics and Gynaecology Research 36, 1071-1074.

7. Tsunoda A, et al. (2016) Survey of electric bidet toilet use among community dwelling Japanese people and correlates for an itch on the anus. Environmental Health and Preventive Medicine 21, 547-553.

8. Kohdaira T (2009) Correlation of recurrent cystitis and using of toilet seat with shower unit. Japanese Journal of Urological Surgery 22, 1217-1222. 
9. Hays RD, Liu H and Kapteyn A (2015) Use of internet panels to conduct surveys. Behavior Research Methods 47, 685-690.

10. Remillard ML, et al. (2014) Systematic review of the use of online questionnaires of older adults. Journal of the American Geriatrics Society 62, 696-705.

11. Yasunaga $\mathbf{H}$, Ide $\mathbf{H}$, Imamura $\mathbf{T}$ and Ohe $\mathbf{K}$ (2006) Medical research using internet questionnaire in Japan. Nihon Koshu Eisei Zasshi 53, 4050, (in Japanese).
12. Ministry of Education, Culture, Sports, Science and Technology, Japan. Statistics, Overview, Enrollment and Advancement Rate. 2016. Available at http://www.mext.go.jp/en/publication/statistics/title01/detail01/1373636. htm\#06 (Accessed 20 September 2017).

13. van Gelder MM, Bretveld RW and Roeleveld N (2010) Web-based questionnaires: the future in epidemiology? American Journal of Epidemiology 172, 1292-1298. 